\title{
Quantum-electrodynamical parametric instability in the incoherent photon gas
}

\author{
Yunliang Wang, ${ }^{1}$ P. K. Shukla, ${ }^{1,2}$ and B. Eliasson ${ }^{1}$ \\ ${ }^{1}$ International Centre for Advanced Studies in Physical Sciences and Institute for Theoretical Physics, \\ Faculty of Physics and Astronomy, Ruhr University Bochum, D-44780 Bochum, Germany \\ ${ }^{2}$ Department of Mechanical and Aerospace Engineering and Center for Energy Research, \\ University of California San Diego, La Jolla, California 92093, USA
}

(Received 11 December 2012; published 25 February 2013)

\begin{abstract}
We present a theory for the quantum-electrodynamical (QED) parametric scattering instability of an intense photon pulse in an incoherent radiation background. The pump electromagnetic (EM) wave can decay into a scattered daughter EM wave and an acousticlike wave due to the QED vacuum polarization nonlinearity. By a linear instability analysis we obtain a nonlinear dispersion relation for the growth rate of the scattering instability. The nonlinear QED scattering instability can give rise to the exchange of orbital angular momentum between intense Laguerre-Gaussian mode photon pulses and the two daughter waves, which may be a useful method to detect the highly energetic photon gases existing in the vicinity of rotating dense bodies in the Universe, such as pulsars and magnetars. The observation of the scattered waves may reveal information about the twisted acoustic waves in the incoherent photon gas.
\end{abstract}

DOI: 10.1103/PhysRevE.87.023105

PACS number(s): 52.35.Mw, 52.40.Db

\section{INTRODUCTION}

The quantum-electrodynamical (QED) photon-photon scattering effects can be expressed through the effective-field theory represented by the Heisenberg-Euler Lagrangian [1], which gives a nonlinear term in the Maxwell's equation that is analogous to self-interaction effects in classic nonlinear optics. Similar to the classic four-wave mixing process, the quantum vacuum effect can generate photons with new frequencies by four-wave interaction due to elastic photonphoton scattering [2]. Recently the matterless double slit was suggested to observe the photon-photon scattering and to realize the controlling of light with light $[3,4]$. The QED corrections can give rise to single photon effects, such as vacuum birefringence [5], photon splitting [6], and lensing effect in strong magnetic fields, as well as collective nonlinear excitations, such as self-focusing of photons, the formation of solitons, generation of harmonic waves, etc. The photon acceleration associated with collective photon interactions has been explored by considering a test photon immersed in a modulated radiation background [7]. These strong-field QED effects have also attracted much attention due to their important implications in the laboratory and astrophysics [8].

Since the lowest order QED effects are proportional to the fine-structure constant squared, the correction term will be important in the presence of high-amplitude electromagnetic fields when they are comparable to the Schwinger critical field $\left(E_{\text {crit }}=10^{16} \mathrm{~V} / \mathrm{cm}\right)$. The available intense laser pulses now reach $10^{22} \mathrm{~W} / \mathrm{cm}^{2}$ (corresponding to an electric field $\sim 10^{12} \mathrm{~V} / \mathrm{cm}$ ) [9] with the help of chirped pulse amplification techniques, and the next generation of lasers may reach up to $10^{26} \mathrm{~W} / \mathrm{cm}^{2}$ (corresponding to $\sim 10^{14} \mathrm{~V} / \mathrm{cm}$ ) [10]. It is expected that the effects of QED in the next generation laser-plasma systems will be important $[8,11]$ and that the nonlinear vacuum effect of elastic photon-photon scattering may be detectable in a plasma channel [12]. The x-ray free-electron lasers (XFEL) [13] are expected to make the quantum vacuum directly accessible for observations, where pair creation is predicted to take place [14].

In fact, extreme conditions are thought to exist in astrophysical settings such as pulsars and magnetars $[15,16]$. In magnetars, the energy densities of the photon gas could be 20-22 orders of magnitude larger than that of the cosmic microwave background, where the energy density is $2.6 \times$ $10^{5} \mathrm{eV} / \mathrm{m}^{3}$ [17]. Hence, one can expect that QED effects will strongly influence nonlinear interactions of an incoherent photon gas with $\gamma$-ray bursts, which are among the most intense pulses in the Universe [18]. Marklund et al. [17] presented a Zakharov-like system of equations describing the nonlinear interactions between intense photon pulses and an incoherent photon gas, where the QED nonlinearity of intense photon pulses drive acousticlike perturbations in the radiation background. It is found that the QED nonlinear effect can give rise to the collapse of intense pulses and the formation of photonic solitons $[19,20]$. The intense photon pulses undergo splitting in due course of time, and wedges are developed in the incoherent photon gas [21].

In this paper we present an investigation of the QED scattering instability of intense photon pulses propagating in the bath of an incoherent photon gas, which is similar to the three-wave process in nonlinear optics. We use the Zakharov-like system [17] to model the scattering process with the help of WKB approximation as well as the energy and momentum conservation. Furthermore, we present possible application of the QED scattering instability to astrophysical settings. The basic equations support Laguerre-Gaussian (LG) mode solutions. The photon orbital angular momentum (OAM) carried by LG states has been used as a method to study the radio sources in astrophysics [22]. The OAM can encode information [23] and give us a new radar communication concepts and methodologies [24], and can help to probe the existence of magnetic monopoles [25]. We expect that our results may be useful to detect information about the incoherent high-energy photon gas in astrophysics settings. 


\section{QED SCATTERING INSTABILITIES}

The photon QED self-interaction can be formulated by the Heisenberg-Euler Lagrangian density [1] $L=\epsilon_{0} F+$ $\kappa \epsilon_{0}^{2}\left[4 F^{2}+7 G^{2}\right]$, where $F=\left(E^{2}-c^{2} B^{2}\right) / 2$ and $G=c \mathbf{E} \cdot \mathbf{B}$, where $\kappa=2 \alpha^{2} \hbar^{3} / 45 m_{e}^{4} c^{5}$ is the coupling constant, $\alpha=$ $e^{2} / 4 \pi \epsilon_{0} \hbar c$ is the fine-structure constant, $\hbar$ is Planck's constant divided by $2 \pi, m_{e}$ is the electron rest mass, $c$ is the speed of light in vacuum, and $\epsilon_{0}$ is the vacuum permittivity. The last two terms in the Heisenberg-Euler Lagrangian density represent the vacuum polarization and magnetization, respectively. Since higher order (in $\alpha$ ) terms are omitted in the Heisenberg-Euler Lagrangian density, the QED modified Maxwell equations are valid only for $|\mathbf{E}| \ll E_{\text {crit }}$ and $c|\mathbf{B}| \ll E_{\text {crit }}$. The dispersion relation for intense photon pulses interacting with an intense background radiation gas with the energy density $\mathcal{E}$ can be written as $\omega \approx c|\mathbf{k}|(1-2 \lambda \mathcal{E})$ [8], where $\lambda=\lambda_{ \pm}$with $\lambda_{+}=14 \kappa$ and $\lambda_{-}=8 \kappa$ standing for two different polarization states of the photons. Here $\omega$ and $\mathbf{k}$ are the frequency and wave vector of intense coherent photon pulses. The energy density of the radiation can be written as $\mathcal{E}=\mathcal{E}_{0}+\mathcal{E}_{g}$ in the presence of propagating intense photon pulses. The perturbed energy density $\mathcal{E}_{g}$ is much smaller than the equilibrium value $\mathcal{E}_{0}$. By using the dispersion relation and the standard method for a slowly varying envelope, based on the MarklundBrodin-Stenflo equation [17], the QED nonlinear interaction of coherent photon pulses with an incoherent photon gas can be modeled by

$$
\begin{gathered}
i\left(\frac{\partial}{\partial t}+c \widehat{\mathbf{k}}_{p} \cdot \nabla\right) E_{p}+\frac{c}{2 k_{p}} \nabla_{\perp}^{2} E_{p}=Q_{1} \mathcal{E}_{g} E_{s}, \\
i\left(\frac{\partial}{\partial t}+c \widehat{\mathbf{k}}_{s} \cdot \nabla\right) E_{s}+\frac{c}{2 k_{s}} \nabla_{\perp}^{2} E_{s}=Q_{2} E_{p} \mathcal{E}_{g}^{*}, \\
i\left(\frac{\partial}{\partial t}+V \widehat{\mathbf{k}}_{g} \cdot \nabla\right) \mathcal{E}_{g}+\frac{V}{2 k_{g}} \nabla_{\perp}^{2} \mathcal{E}_{g}=Q_{3} E_{p} E_{s}^{*},
\end{gathered}
$$

where the coupling coefficients are $Q_{1}=-2 \lambda c k_{p} / 3, Q_{2}=$ $-2 \lambda c k_{s} / 3$, and $Q_{3}=-4 V k_{g} \lambda \epsilon_{0} \mathcal{E}_{0} / 3$, which come from the QED vacuum polarization effect. Here we have assumed the electromagnetic (EM) waves to be of the form $E_{p, s} \exp \left(i k_{p, s}-\right.$ $\left.i \omega_{p, s}\right)+$ c.c. (c.c. standing for complex conjugate) and the acoustic perturbations in a photon gas $\mathcal{E}_{g} \exp \left(i k_{g}-i \omega_{g}\right)+$ c.c., with slowly varying amplitudes $E_{p}$ and $E_{s}$ standing for the amplitudes of the pump photon pulse and scattered waves, respectively. The electric and magnetic fields are $\mathbf{E}_{p, s}=E_{p, s} \widehat{\mathbf{e}}_{p, s}$ and $\mathbf{B}_{p, s}=E_{p, s} \widehat{\mathbf{k}}_{p, s} \times \widehat{\mathbf{e}}_{p, s} / c$, respectively, where $\widehat{\mathbf{e}}_{p, s}$ is the unit vector in the direction of $\mathbf{E}_{p, s}$. The asterisk denotes complex conjugate. In the derivation above, we also used the standard eikonal representation and the WKB approximation $\left|\partial E_{p, s} / \partial t\right| \ll\left|\omega_{p, s} E_{p, s}\right|,\left|\partial \mathcal{E}_{g} / \partial t\right| \ll\left|\omega_{g} \mathcal{E}_{g}\right|$, $\left|\widehat{\mathbf{k}}_{p, s} \cdot \nabla E_{p, s}\right| \ll\left|k_{p, s} E_{p, s}\right|$, and $\left|\widehat{\mathbf{k}}_{g} \cdot \nabla \mathcal{E}_{g}\right| \ll\left|k_{g} \mathcal{E}_{g}\right|$. The unit vectors are defined as $\widehat{\mathbf{k}}_{p} \equiv \mathbf{k}_{p} / k_{p}, \widehat{\mathbf{k}}_{s} \equiv \mathbf{k}_{s} / k_{s}$, and $\widehat{\mathbf{k}}_{g} \equiv$ $\mathbf{k}_{g} / k_{g}$. The differential operators $\nabla_{\perp}^{2}$ are $\nabla^{2}-\left(\widehat{\mathbf{k}}_{p, s, g} \cdot \nabla\right)^{2}$ in Eqs. (1)-(3), respectively.

Equations (1)-(3) can be used to investigate the parametric scattering instability where an intense EM wave $E_{p}$ decays into a scattered EM wave $E_{s}$ and an acousticlike wave $E_{g}$ in the background intense radiation gas. In the parametric process, the energy and momentum conservation relations are $\omega_{p}=$ $\omega_{s}+\omega_{g}$ and $\mathbf{k}_{p}=\mathbf{k}_{s}+\mathbf{k}_{g}$ with $\omega_{p, s, g}$ and $\mathbf{k}_{p, s, g}$ representing the frequencies and wave vectors of the pump EM wave, scattered EM waves, and acousticlike waves, respectively. For the background photon gas, the directions of the photons in the gas are random, and the acousticlike waves have the effective acoustic speed $V=c / \sqrt{3}$ in the averaged fluid description of the photon gas. The dynamical equation, which has been derived from the radiation hydrodynamic equations, for the acousticlike perturbation (see Refs. $[8,17,19]$ ), are driven by the QED nonlinearity of the intense EM pulses.

We first present a linear instability analysis of the QED scattering process. For the parametric approximation, we consider a homogeneous intense EM wave $E_{p}$, with $E_{p} \gg$ $E_{s}$ and $\epsilon_{0}\left|E_{p}\right|^{2} \mid \gg \mathcal{E}_{g}$. The scattered EM wave is $E_{s}=$ $\widehat{E}_{s} \exp (i \mathbf{K} \cdot \mathbf{r}-i \Omega t)+$ c.c., and the acoustic wave is $\mathcal{E}_{g}=$ $\widehat{\mathcal{E}}_{g} \exp (-i \mathbf{K} \cdot \mathbf{r}+i \Omega t)+$ c.c., with the scattered wave frequency $\Omega$ and vector $\mathbf{K}$. Inserting these Fourier representations into Eqs. (1)-(3) and separating different Fourier modes and eliminating the Fourier coefficients, we obtain the nonlinear dispersion relation

$$
\begin{aligned}
& \left(\Omega-c K_{\|}+\frac{c K_{\perp}^{2}}{2 k_{s}}\right)\left(\Omega-V K_{\|}-\frac{V K_{\perp}^{2}}{2 k_{g}}\right) \\
& +Q_{2} Q_{3}\left|E_{p}\right|^{2}=0
\end{aligned}
$$

where $K_{\|}$and $K_{\perp}$ are the parallel and perpendicular components of the wave vector $\mathbf{K}$, respectively. The growth-rate $\gamma$ of the instability (with $\Omega=\Omega_{r}+i \gamma$ ) is

$$
\begin{aligned}
\gamma= & {\left[-\frac{1}{4}\left(c K_{\|}-\frac{c K_{\perp}^{2}}{2 k_{s}}-V K_{\|}-\frac{V K_{\perp}^{2}}{2 k_{g}}\right)^{2}\right.} \\
& \left.+Q_{2} Q_{3}\left|E_{p}\right|^{2}\right]^{1 / 2} .
\end{aligned}
$$

For the parallel case $K_{\|}=K$ and $K_{\perp}=0$, the growth rate is $\gamma=\left[Q_{2} Q_{3}\left|E_{p}\right|^{2}-(c-V)^{2} K_{\|}^{2} / 4\right]^{1 / 2}$ for $K<$ $4 \lambda\left|E_{p}\right|\left(c V k_{s} k_{g} \epsilon_{0} \mathcal{E}_{0}\right)^{1 / 2} / 3(c-V)$.

Next, we use numerical simulations to study the nonlinear evolution of the QED scattering processes. We use a pseudospectral method for spatial derivatives with periodic boundary conditions and the standard fourth-order Runge-Kutta method for time stepping. Our numerical results of Eqs. (1)-(3) are displayed in Fig. 1. The initial value of the homogeneous pump pulse is $\lambda \sqrt{\epsilon_{0} \mathcal{E}_{0}} E_{p}=\sqrt{0.02}$ at $t=0$. A low-amplitude noise of the order $10^{-4}$ was used as initial conditions for the scattering wave $\lambda \sqrt{\epsilon_{0} \mathcal{E}_{0}} E_{S}$ and acoustic wave $\lambda \mathcal{E}_{g}$ to give a seed for any instabilities excited by the QED nonlinearity. We used the wave numbers $k_{s}=0.9 k_{p}$ and $k_{g}=0.1 k_{p}$. The results in Fig. 1 shows that initially the scattered EM waves and acousticlike waves grow exponentially. At time $k_{p} c t=270$, the pump wave is depleted and the amplitudes of the scattered daughter waves and acousticlike waves reach a maximum. The lower-right panel in Fig. 1 shows the time evolution of the amplitudes of $E_{p}, E_{s}$, and $\mathcal{E}_{g}$ at $z=0$, exhibiting a predator-prey-like behavior where the wave energy oscillates between the different wave modes.

Let us consider the multidimensional instability of the QED scattering process. First, we neglect the time derivatives and coupling terms in the right-hand side of Eqs. (1)-(3), to obtain 

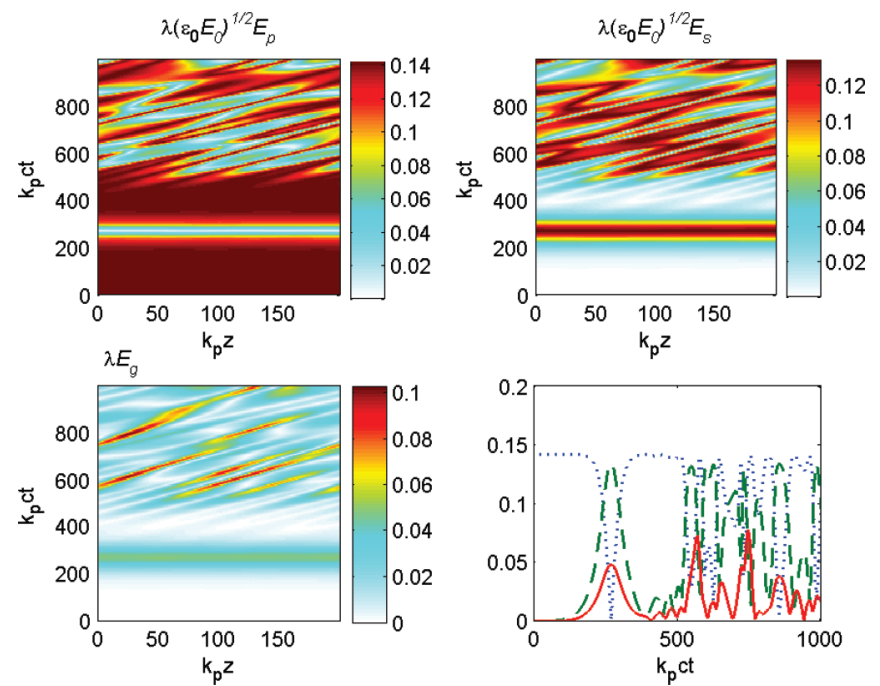

FIG. 1. (Color online) The dynamics of the QED scattering instability of intense EM pulses in an incoherent photon gas. The pump pulse $E_{p}$ decays into scattered waves $E_{s}$ and acousticlike waves $\mathcal{E}_{g}$. The parameters are $k_{s}=0.9 k_{p}$ and $k_{g}=0.1 k_{p}$. At time $t=270 / k_{p} c$, the pump $E_{p}$ depletes and $E_{s}$ and $E_{g}$ have amplitude maxima. In the lower right panel, the amplitudes of the $E_{p}$ (dotted curve), $E_{d}$ (dashed curve), and $\mathcal{E}_{g}$ (solid curve) at $z=0$ are plotted versus time.

the paraxial equations

$$
\begin{gathered}
i \frac{\partial E_{p}}{\partial z}+\frac{1}{2 k_{p}} \nabla_{\perp}^{2} E_{p}=0, \\
i \frac{\partial E_{s}}{\partial z}+\frac{1}{2 k_{s}} \nabla_{\perp}^{2} E_{s}=0, \\
i \frac{\partial \mathcal{E}_{g}}{\partial z}+\frac{1}{2 k_{g}} \nabla_{\perp}^{2} \mathcal{E}_{g}=0,
\end{gathered}
$$

where we have assumed that the three waves propagate primarily along the $z$ direction. These paraxial equations can be expressed in cylindrical coordinates $r, \varphi, z$ with $\nabla_{\perp}^{2}=$ $(1 / r)(\partial / \partial r)(r \partial / \partial r)+\left(1 / r^{2}\right) \partial^{2} / \partial \varphi^{2}$, and solved in terms of LG modes as [26-28]

$$
\begin{aligned}
E_{p} & =\widetilde{E}_{p}(z) F_{p_{1}, l_{1}}(r, z) e^{i l_{1} \varphi}, \\
E_{s} & =\widetilde{E}_{s}(z) F_{p_{2}, l_{2}}(r, z) e^{i l_{2} \varphi}, \\
\mathcal{E}_{g} & =\widetilde{\mathcal{E}}_{g}(z) F_{p_{3}, l_{3}}(r, z) e^{i l_{3} \varphi},
\end{aligned}
$$

where $F_{p_{j}, l_{j}}(r, z)$, with $j=1,2,3$, are the LG functions, given by

$$
F_{p_{j}, l_{j}}(r, z)=\frac{1}{2 \sqrt{\pi}}\left[\frac{\left(p_{j}+l_{j}\right) !}{p_{j} !}\right]^{1 / 2} X^{\left|l_{j}\right|} L_{p_{j}}^{\left|l_{j}\right|}(X) e^{-\frac{X}{2}} .
$$

Here $X=r^{2} / w_{j}^{2}$ and $w_{j} \equiv w_{j}(z)$ are the beam waists of the EM waves and acousticlike waves, and the associated Laguerre polynomials $L_{p_{j}}^{\left|l_{j}\right|}(X)$ are defined by the Rodriguez formula $L_{p_{j}}^{\left|l_{j}\right|}(X)=\left(X^{l_{j}} p_{j} !\right)^{-1} \exp (X) d^{p}\left[X^{l_{j}+p_{j}} \exp (-X)\right] / d X^{p}, \quad p_{j}$ and $l_{j}$ are the radial and angular mode numbers of the LG photon pulse, respectively, $\varphi$ is the azimuthal angle, and the azimuthal modes $l_{j}$ are the eigenmodes of the angular momentum operator $L^{z}$ [28]. Although acoustic waves (such as electron plasma waves and ion-acoustic waves) have no intrinsic eigenmodes of the angular momentum in contrast to the EM pulses, the corresponding phonons or plasmons can also carry OAM [26]. The LG-mode phonons can be excited by the stimulated Raman or Brillouin scattering of laser beams in plasmas [29]. The daughter scattering waves can also contain information carried by the pump LG pulse in the plasma [27]. Here we derive the solutions with LG modes of the paraxial equations for the acousticlike waves exited by the QED nonlinearity in the incoherent photon gas background. The solution of the acousticlike wave with LG modes can be written as $\mathcal{E}_{g}=\widetilde{\mathcal{E}}_{g} F_{p_{3}, l_{3}}(r, z) \exp \left(i l_{3} \varphi\right) \exp \left(i k_{g} z-i \omega_{g} t\right)+$ c.c.

We next investigate numerically the temporal evolution and exchange of OAM between the pump and the scattered EM waves and acousticlike waves in a photon gas. For this purpose, we use the LG solution [cf. Eq. (7)] as an initial condition for $E_{p}$ in our simulation. The other parameters are $k_{s}=0.7 k_{p}$ and $k_{g}=0.3 k_{p}$. The initial pump LG wave is $\lambda \sqrt{\epsilon_{0} \mathcal{E}_{0}} E_{p}=$ $\sqrt{0.002} \sqrt{3 / 4 \pi} r \exp (-r / 2)\left(3-3 r+r^{2} / 2\right)$ with $r=\left(x^{2}+\right.$ $\left.y^{2}\right)^{1 / 2}$, obtained by taking $p_{1}=2$ and $l_{1}=1$ in the LG functions. A low-amplitude noise of the order $10^{-4}$ is used as an initial condition for $\lambda \sqrt{\epsilon_{0} \mathcal{E}_{0}} E_{s}$ and $\lambda \mathcal{E}_{g}$, to give a seed for any instability. Initially, the scattered wave and acousticlike wave grow exponentially and exhibit the signatures of LG modes. We confirm the assumption of the exchange of OAM between the pump EM wave and two daughter EM waves. Furthermore, the energy conservation is also confirmed by our numerical simulations. It should be mentioned that the energy will exchange between the EM pump wave and the two daughter EM waves due to the QED nonlinearity. To evaluate the growth rate of the instability of QED scattering, we suppose that the amplitudes depend on time and rewrite the nonlinearly coupled equations (1)-(3) as $i \partial \widetilde{E}_{p} / \partial t=\widetilde{Q}_{1} \widetilde{\mathcal{E}}_{g} \widetilde{E}_{s}, i \partial \widetilde{E}_{s} / \partial t=$ $\widetilde{Q}_{2} \widetilde{\mathcal{E}}_{g}^{*} \widetilde{E}_{p}$, and $i \partial \widetilde{\mathcal{E}}_{g} / \partial t=\widetilde{Q}_{3} \widetilde{E}_{p} \widetilde{E}_{s}^{*}$, where the coefficients are $\widetilde{Q}_{1}=2 \pi Q_{1} R, \widetilde{Q}_{2}=2 \pi Q_{2} R$, and $\widetilde{Q}_{3}=2 \pi Q_{3} R$, and the quantity $R \equiv R(z)=\int_{0}^{\infty} F_{p_{1}, l_{1}} F_{p_{1}, l_{1}} F_{p_{1}, l_{1}} r d r$ [26]. We consider the parametric instability and assume that $\partial \widetilde{E}_{p} / \partial t \simeq$ 0 . Henceforth, we can derive

$$
\begin{aligned}
& \frac{\partial^{2} \widetilde{E}_{s}}{\partial t^{2}}-\widetilde{Q}_{2} \widetilde{Q}_{3}\left|\widetilde{E}_{p}\right|^{2} \widetilde{E}_{s}=0, \\
& \frac{\partial^{2} \widetilde{\mathcal{E}}_{g}}{\partial t^{2}}-\widetilde{Q}_{2} \widetilde{Q}_{3}\left|\widetilde{E}_{p}\right|^{2} \widetilde{\mathcal{E}}_{g}=0 .
\end{aligned}
$$

From Eq. (9) one can easily obtain the solutions as $\widetilde{E}_{s}(z, t)=\widetilde{E}_{s}(z, 0) \exp (\tilde{\gamma} t)$ and $\widetilde{\mathcal{E}}_{g}(z, t)=\widetilde{\mathcal{E}}_{g}(z, 0) \exp (\widetilde{\gamma} t)$ with the growth rate $\tilde{\gamma}=\left(\widetilde{Q}_{2} \widetilde{Q}_{3}\right)^{1 / 2}\left|\widetilde{E}_{p}\right|$. Apparently, the derived growth rate is valid if the nonlinearity is very weak. As illustrated in Figs. 1 and 2, the growth rate of the scattered wave is different from that of the acoustic perturbation, which is due to the fact that the QED nonlinearity is not weak. The numerical results show that the incident pump EM pulse can excite daughter EM waves and acousticlike waves of a photon gas with different LG modes. Furthermore, one can determine the LG modes of the acoustic perturbations in the photon gas by checking the states of scattered daughter EM waves, which can be guided by the growth rate $\tilde{\gamma}$ due to the orthogonality relations of the LG functions. Thus, our results may have potential applications in astronomy. Harwit first studied the OAM for the astronomy application [30]. Recently, Tamburini 

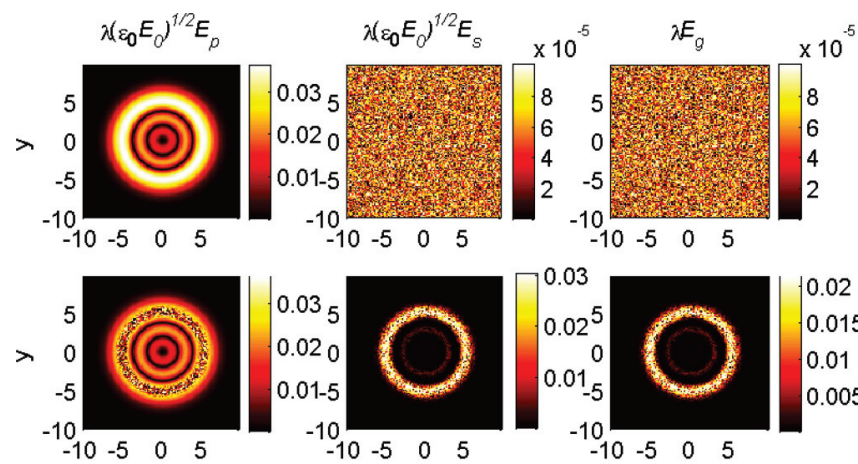

$\begin{array}{llll}-10 & -5 & 0 & 5\end{array}$
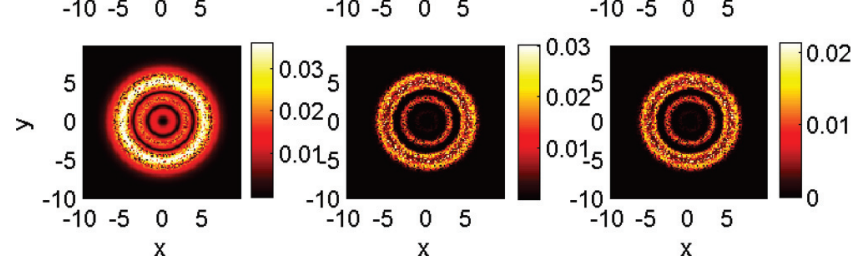

FIG. 2. (Color online) The dynamics of OAM exchange due to the QED scattering instability between the pump EM wave $\lambda \sqrt{\epsilon_{0} \mathcal{E}_{0}} E_{p}$ and scattered EM waves $\lambda \sqrt{\epsilon_{0} \mathcal{E}_{0}} E_{s}$ as well as acousticlike waves $\lambda \mathcal{E}_{g}$ at different times $k_{p} c t=0,600$, and 800 (upper to lower panels). The parameters are $k_{s}=0.7 k_{p}$ and $k_{g}=0.3 k_{p}$.

et al. [31] suggest that on Earth, the astronomers can set up the best existing telescope to detect and measure light that has been twisted by rotating massive black holes. Since the rotation is common for astrophysical objects in the Universe, we may expect that the incoherent photon gas existing in the vicinity of pulsars and magnetars may have acoustic LG modes twisted by the new relativistic effect of space-time wrap up of rotating pulsars or magnetars. Accordingly, from the OAM states of the scattering EM waves excited by the QED nonlinearity we can obtain information about the massive astrophysical objects.

\section{SUMMARY AND CONCLUSIONS}

In summary, we have studied stimulated scattering instabilities of photon pulses in the bath of an incoherent photon gas. Specifically, the focus has been on the parametric instability caused by the QED nonlinearity. The expression for the growth rate has been obtained. The numerical results of the governing Eqs. (1)-(3) reveal that the pump EM wave decays into daughter EM waves and an acousticlike perturbation. At a certain time, the pump EM wave depletes and after that begins to exhibit a nonlinear oscillatory phase, similar to a predator-prey system.

We also present an important application of the QED scattering to astrophysical settings. By neglecting the time derivatives and nonlinear coupling term, we obtain LG mode solutions for the pump EM waves, scattering EM waves, and acoustic waves. The exchange of OAM between the pump EM wave and the daughter EM wave and acousticlike wave has been confirmed by numerical simulations. By considering the orthogonality relations of the LG functions, the scattered EM waves may carry information about the incoherent photon gas, which can be used to detect the astrophysical objects as the OAM states can be twisted by the space-time wrap up of rotating pulsars and magnetars.

\section{ACKNOWLEDGMENTS}

One of the authors (Y.W.) thanks financial support of NSFC (No. 11104012) and the Fundamental Research Funds for the Central Universities (No. FRF-TP-09-019A, No. FRF-BR-11031B).
[1] W. Heisenberg and H. Euler, Z. Phys. 98, 714 (1936).

[2] E. Lundström, G. Brodin, J. Lundin, M. Marklund, R. Bingham, J. Collier, J. T. Mendonça, and P. Norreys, Phys. Rev. Lett. 96, 083602 (2006).

[3] B. King, A. Di Piazza, and C. H. Keitel, Nat. Photon. 4, 92 (2010).

[4] B. King, A. Di Piazza, and C. H. Keitel, Phys. Rev. A 82, 032114 (2010).

[5] P. Berceau, M. Fouché, R. Battesti, and C. Rizzo, Phys. Rev. A 85, 013837 (2012).

[6] G. Brodin, M. Marklund, B. Eliasson, and P. K. Shukla, Phys. Rev. Lett. 98, 125001 (2007).

[7] J. T. Mendonça, M. Marklund, P. K. Shukla, and G. Brodin, Phys. Lett. A 359, 700 (2006).

[8] M. Marklund and P. K. Shukla, Rev. Mod. Phys. 78, 591 (2006).

[9] V. Yanovsky, V. Chvykov, G. Kalinchenko, P. Rousseau, T. Planchon, T. Matsuoka, A. Maksimchuk, J. Nees, G. Cheriaux, G. Mourou, and K. Krushelnick, Opt. Express 16, 2109 (2008).

[10] A. Di Piazza, C. Müller, K. Z. Hatsagortsyan, and C. H. Keitel, Rev. Mod. Phys. 84, 1177 (2012).

[11] G. A. Mourou, T. Tajima, and S. V. Bulanov, Rev. Mod. Phys. 78, 309 (2006)
[12] B. Shen, M. Y. Yu, and X. Wang, Phys. Plasmas 10, 4570 (2003).

[13] E. Hand, Nature (London) 461, 708 (2009).

[14] C. D. Roberts, S. M. Schmidt, and D. V. Vinnik, Phys. Rev. Lett. 89, 153901 (2002).

[15] M. G. Baring and A. K. Harding, Astrophys. J. 507, L55 (1998).

[16] M. G. Baring and A. K. Harding, Astrophys. J. 547, 929 (2001).

[17] M. Marklund, G. Brodin, and L. Stenflo, Phys. Rev. Lett. 91, 163601 (2003).

[18] T. Piran, Phys. Rep. 314, 575 (1999).

[19] P. K. Shukla and B. Eliasson, Phys. Rev. Lett. 92, 073601 (2004).

[20] M. Marklund, B. Eliasson, and P. K. Shukla, JETP Lett. 79, 262 (2004).

[21] P. K. Shukla, M. Marklund, D. D. Tskhakaya, and B. Eliasson, Phys. Plasmas 11, 3767 (2004).

[22] B. Thidé, H. Then, J. Sjöholm, K. Palmer, J. Bergman, T. D. Carozzi, Ya. N. Istomin, N. H. Ibragimov, and R. Khamitova, Phys. Rev. Lett. 99, 087701 (2007).

[23] A. Watsons, Science 296, 2316 (2002).

[24] G. Gibson, J. Courtial, M. J. Padgett, M. Vasnetsov, V. Pas'ko, S. M. Barnett, and S. Franke-Arnold, Opt. Express 12, 5448 (2004). 
[25] N. H. Ibragimov, R. Khamitova, and B. Thidé, J. Math. Phys. 48, 053523 (2007).

[26] J. T. Mendonça, B. Thidé, and H. Then, Phys. Rev. Lett. 102, 185005 (2009); J. T. Mendonça, Plasma Phys. Controlled Fusion 54, 124031 (2012).

[27] P. K. Shukla, B. Eliasson, and L. Stenflo, Phys. Rev. E 86, 016403 (2012).
[28] L. Allen, M. W. Beijersbergen, R. J. C. Spreeuw, and J. P. Woerdman, Phys. Rev. A 45, 8185 (1992).

[29] M. K. Ayub, S. Ali, and J. T. Mendonça, Phys. Plasmas 18, 102117 (2011).

[30] M. Harwit, Astrophys. J. 597, 1266 (2003).

[31] F. Tamburini, B. Thidé, G. Molina-Terriza, and G. Anzolin, Nat. Phys. 7, 195 (2011). 